\title{
Articulaciones entre desigualdades, aprendizajes y tecnologías digitales: un recorrido por conceptos clave
}

\author{
A review of the main concepts on inequalities, learning and \\ digital technologies
}

\section{Magdalena Lemus}

magdalenalemus.2@gmail.com

Centro Interdisciplinario de Metodología de las

Ciencias Sociales. Instituto de Investigaciones en Humanidades y Ciencias Sociales (IdIHCS CONICET). Universidad Nacional de La Plata, Argentina

\section{Recepción: 15 Mayo 2020 \\ Aprobación: 27 Octubre 2020 \\ Publicación: 01 Febrero 2021}

Cita sugerida: Lemus, M. (2021). Articulaciones entre desigualdades, aprendizajes y tecnologías digitales: un recorrido por conceptos clave. Cuestiones de Sociología, 24, e118. https://doi.org/10.24215/23468904e118

\begin{abstract}
Resumen: El cruce entre tecnologías y desigualdades ha generado diversos debates y posicionamientos, permaneciendo vigente conforme cambian los contextos, las tecnologías y las iniciativas de los Estados y organizaciones para afrontar las desigualdades. En este trabajo, presentamos discusiones y conceptualizaciones teóricas que se han elaborado en torno al vínculo entre desigualdades y tecnologías digitales a lo largo de las últimas dos décadas. A su vez, analizamos los corrimientos que ha experimentado este campo temático, involucrando nuevas dimensiones de análisis y pasando del foco en el acceso a la incorporación de los interrogantes por las calidades, las prácticas y las alfabetizaciones. Para adelantar algunas conclusiones, podemos señalar que el análisis que aquí presentamos da cuenta del dinamismo con que el campo académico ha buscado comprender los diversos procesos de cambio que han tenido lugar a partir de la participación de las tecnologías digitales en diversas esferas de nuestras sociedades. En este sentido, identificamos una variedad de conceptos que, con distinto alcance y popularidad, han intentado describir nuevas prácticas y significados y su intersección con estructuras y procesos sociales de larga data.
\end{abstract}

Palabras clave: Desigualdades, Tecnologías digitales, Alfabetizaciones.

\begin{abstract}
In the last two decades information and communication technologies as well as digital technologies became relevant issues for social sciences. The linkage between access and use of these technologies and social inequalities brought up important theoretical and political debates and continue to be major issues for governments and organizations that intend to tackle poverty and inequalities. This paper examines the theoretical frameworks addressing the relationship between digital technologies and inequalities. We focus on concepts used to describe differences and inequalities on access, use and literacies. We managed to identify a wide array of terms and ideas which were proposed as conceptual tools to understand how inequalities shape our skills to use digital technologies in diverse and meaningful ways. Even though some of these concepts have become more or less popular, and others remain constrained to scholarly research, their emergence suggest a dynamic area of study
\end{abstract}


where scholars from different regions are seeking for a better understanding of a challenging and vertiginous and phenomenon.

Keywords: Inequalities, Digital technologies, Literacies.

\section{Introducción}

$\mathrm{La}^{1}$ pregunta sobre los modos en que se producen y reproducen las desigualdades se ha constituido a lo largo del tiempo como una de las temáticas medulares de las ciencias sociales. Las respuestas se han erigido sobre la base de distintas miradas en torno al individuo y la sociedad y, por lo tanto, sobre el origen de las desigualdades (Reygadas, 2008). En los últimos años, hemos presenciado el resurgimiento de las desigualdades como problemática sociológica y social. Los modos de comprenderlas, estudiarlas y transformarlas han sido nuevamente puestos en el centro de la escena tanto en la producción académica latinoamericana y mundial, como en el debate político y en las reivindicaciones de distintos actores sociales, desde donde se ha legitimado la lucha por la igualdad (Kessler, 2014). Junto a esto, se han multiplicado las voces que señalan que las desigualdades deben ser abordadas en sus múltiples dimensiones (Saraví, 2015), atendiendo no solo a los factores económicos sino también a los procesos culturales por medio de los cuales este fenómeno se produce, reproduce y transforma (Lamont, Beljean y Clair, 2014).

Las tecnologías digitales y los modos en que son apropiadas han merecido una atención creciente en el campo de la sociología y otras ciencias sociales y humanidades. El cruce entre tecnologías digitales ${ }^{2}$ y desigualdades ha generado acalorados debates y posturas antagonistas, manteniéndose vigente conforme cambian los contextos, las tecnologías y las iniciativas de los Estados y otras organizaciones para afrontar las desigualdades. El acceso y la capacidad para usar tecnologías digitales (en adelante, TD) ha sido destacada como un aspecto fundamental en las sociedades contemporáneas, tanto para el acceso a la información y la comunicación como para el ejercicio de la ciudadanía y la participación en el mercado de trabajo (UNESCO, 2018).

De acuerdo con Reygadas, en torno a la articulación entre desigualdades y tecnologías se han conformado al menos dos polos (Reygadas, 2008). Para algunos, las TD cargan con la responsabilidad de aliviar, disminuir e incluso combatir las desigualdades. Para otros, el acceso a la información y a dispositivos, así como los cambios que estos "pueden provocar", se suman a la larga lista de factores que contribuyen a profundizar y reproducir las desigualdades. Estas miradas han permeado tanto al campo de la investigación social como también han sido la base sobre la cual, durante varias décadas, se han implementado políticas públicas de acceso y alfabetización en TD.

La pandemia de COVID19 y las medidas de aislamiento y distanciamiento social preventivo y obligatorio implementadas para hacerle frente han puesto de relieve, una vez más, la vigencia de las desigualdades relativas a las TD. En el caso de Argentina, la interrupción de la educación formal presencial en todos los niveles educativos y la puesta en marcha de instancias virtuales, junto al traslado de tareas laborales al hogar a través del teletrabajo -en los casos en los 
que fue posible- dejaron al descubierto las dificultades que enfrentan personas de distintas clases sociales, generaciones y géneros para acceder a las TD y utilizarlas con diversos fines. En particular, las discusiones sobre las competencias necesarias para teletrabajar, enseñar y aprender en contextos de pandemia y aislamiento social fueron algunas de las cuestiones que quedaron sobre el tapete y evidenciaron que no solo se trata de acceder materialmente a las TD, cuestión que ha sido largamente señalada por las investigaciones en el tema (Helsper, 2017; Winocur, 2009, entre otras).

Teniendo en cuenta los desafíos que emergieron en este contexto y las temáticas propuestas en el presente dossier, en este trabajo presentamos discusiones y conceptualizaciones teóricas que se han elaborado en torno al vínculo entre desigualdades y tecnologías digitales, atendiendo especialmente a la intersección de estas cuestiones con la dimensión educativa. Para ello, a lo largo del artículo recorremos algunos de los conceptos que han cobrado más notoriedad y destacamos aquellos abordajes que consideramos tienen mayor potencialidad para comprender de qué manera en nuestras sociedades contemporáneas se articulan desigualdades, tecnologías digitales y alfabetizaciones.

Para identificar y caracterizar los diversos enfoques teóricos sobre el tema, llevamos adelante un relevamiento bibliográfico en revistas y libros de referencia en este campo temático. Como distintos autores han señalado (a nivel mundial: Castells, 2008; latinoamericano: Winocur, 2007; y argentino: Benítez Larghi, Aguerre, Calamari, Fontecoba, Moguillansky y Ponce de León, 2011; entre otros), el proceso de expansión de las tecnologías digitales tuvo lugar en sociedades heterogéneas y caracterizadas por múltiples desigualdades sociales, económicas, generacionales, étnicas y de género, teniendo como resultado la configuración de esquemas desiguales de acceso a las tecnologías. Los trabajos relevados corresponden al período 2000-2020, selección que buscó captar el surgimiento de conceptualizaciones en un contexto de masificación inicial a nivel mundial y regional del acceso a distintas TD y su derrotero conforme evolucionaban los niveles de acceso, ${ }^{3}$ las problemáticas y las apropiaciones sociales de las TD. Se relevó bibliografía internacional, regional y local, atendiendo a los conceptos que mayor resonancia han adquirido en el campo académico y también a aquellos que, tal vez con menos popularidad, han buscado incorporar nuevas dimensiones para el análisis.

Para adelantar algunas conclusiones, podemos señalar que el análisis que aquí presentamos da cuenta del dinamismo con que el campo académico ha buscado comprender los diversos procesos de cambio que han tenido lugar a partir de la participación de las TD en diversas esferas de nuestras sociedades. En este sentido, identificamos una variedad de conceptos que, con distinto alcance y popularidad, han intentado describir nuevas prácticas y significados y su intersección con estructuras y procesos sociales de larga data.

\section{De la brecha digital a la relatividad social de las desigualdades digitales}

El recorrido por la literatura especializada pone de relieve la existencia de una serie de términos que, en las últimas dos décadas, se han vuelto centrales 
para el estudio del vínculo entre TD y desigualdades tales como brecha digital, capital informático, pobreza digital, apropiación, entre otros. Entre todos ellos, el de brecha digital ha sido de los que más notoriedad y difusión ha ganado. La preeminencia de este concepto en el mundo académico ha tenido su correlato en el campo de las políticas públicas, en donde la noción se ha instalado con fuerza tanto entre representantes gubernamentales y tomadores de decisiones, como en los discursos de organizaciones no gubernamentales y organismos de crédito y financiamiento.

El término brecha digital comenzó a utilizarse a finales de los años 70 para conceptualizar los modos en que la difusión de las tecnologías se articulaban con la desigualdad social, así como el impacto que estas tenían en el marco de sociedad heterogéneas y desiguales, a las cuales el acceso a las tecnologías llegaba en distintos tiempos y formas. A partir de esta noción se buscó dar cuenta de la existencia de "distancias" en materia de acceso a las TD por parte, en un principio, de distintos países a nivel mundial. De acuerdo con Camacho (2005), en este primer momento, la brecha digital era entendida como la distancia de acceso a las tecnologías digitales, que se relacionaba con las posibilidades de desarrollo de las sociedades. De esta manera, se pensaba que, si se acortaba esa distancia, es decir, si se garantizaba el acceso a las TD, se alcanzaría el desarrollo. Desde esta perspectiva tecno-céntrica, se establecía un vínculo lineal entre acceso a la tecnología y desarrollo, dotando a la primera de cualidades intrínsecamente positivas (Camacho, 2005). Se ocultaban así las profundas desigualdades entre países y regiones que arrojaban distintos esquemas de acceso a las tecnologías arraigados en profundas y diversas inequidades estructurales.

Años más tarde, a la luz de nuevos debates políticos y académicos así como de la expansión de las TD a nivel mundial y la persistencia de desigualdades, la definición de brecha digital fue transformándose, incorporando nuevas dimensiones tales como la condición socioeconómica, el género, la edad, la localización geográfica, las prácticas culturales, entre otras (Camacho, 2005). Así, se comenzó a conceptualizar el vínculo entre las TD y la desigualdad en términos no solo de acceso al equipamiento y la infraestructura (brecha digital de primer orden), sino también en relación a la posesión de habilidades y conocimientos específicos (brecha digital de segundo orden) y a las modalidades y finalidades de uso (brecha digital de tercer orden).

Como ha sostenido Potter (2007), una característica central del concepto de brecha digital es que es "flexible" y que, por lo tanto, puede albergar múltiples significados, así como transformarse conforme cambian las tecnologías (Gunkel, 2003 citado en Potter, 2007). El término ha servido tanto para definir las distintas experiencias entre los/as primeros/as adoptantes de TD y quienes lo hicieron posteriormente, hasta las desigualdades de acceso entre habitantes de países desarrollados y en desarrollo (Potter, 2007). Sin embargo, estas aparentes cualidades son también el eje de las críticas que ha recibido. Por ejemplo, se ha mencionado que el concepto de brecha digital tiende a dividir a la población de forma dicotómica, entre aquellos que tienen y los que no tienen acceso a las TD (Selwyn, 2004). Junto a esto, se ha señalado que uno de los mayores errores es entender a la brecha digital como la causa de la desigualdad de oportunidades socioeconómicas en vez de como un síntoma de esta (Gunkel, 2003 en Potter, 2007). Por su parte, Warschauer (2002) ha destacado que el acceso a las TD 
implica no solo la posibilidad de usar la tecnología sino que hay un conjunto de factores humanos, técnicos, institucionales, educativos y relacionales que influyen en dicho acceso y que deben ser analizados. De lo contrario, se caerá en la creencia de que las desigualdades se subsanan únicamente brindando TD.

Con la vocación de construir interpretaciones que tengan en cuenta las múltiples dimensiones que participan en la articulación entre TD y desigualdad y, a la vez, trasciendan la dicotomía "acceso-no acceso", fueron emergiendo nuevas conceptualizaciones. Por ejemplo, la propuesta desarrollada a partir de la noción de pobreza digital trata de ampliar el foco de indagación hacia los motivos que llevan al acceso/ no acceso y al uso/no uso de las TD y TIC (Barrantes, 2007). Para ello, se contemplan experiencias y situaciones en donde el no acceso y el no uso (que no son tomados como sinónimos) están dados por motivos que no necesariamente implican una carencia económica. La pobreza digital puede ser entendida como "la carencia de bienes y servicios basados en TICs" (Barrantes, 2007, p. 2) y no siempre es producto de la pobreza económica, sino que deben ser considerados todos aquellos elementos y factores que exceden a los materiales y que intervienen para que una persona no acceda y no use estas tecnologías.

De acuerdo con Di Maggio, Hargittai, Celeste y Shafer (2001), la mirada dicotómica "accede-no accede" tuvo utilidad en los primeros momentos de difusión cuando el acceso no estaba muy extendido. Sin embargo, en un contexto de crecimiento del acceso en donde las dimensiones relativas a la calidad comienzan a volverse el sustrato a partir del cual los "beneficios" de las TD se estratifican, los interrogantes deben reformularse. Así, se vuelve necesario redefinir qué se entiende por acceso e indagar en qué es lo que las personas están haciendo y qué pueden hacer cuando se conectan (Di Maggio, Hargittai, Celeste y Shafer, 2001), es decir, preguntarse por las múltiples dimensiones que hacen al uso de las computadoras e Internet y que no están contempladas desde la noción de brecha digital. Por esto, señalan que el concepto desigualdad digital permite conocer mucho mejor las potenciales consecuencias de accesos y usos a Internet diferenciales producto de condiciones socioeconómicas distintas (Di Maggio, Hargittai, Celeste y Shafer, 2001).

En sintonía con la perspectiva mencionada en el párrafo anterior, Selwyn (2004) retoma el enfoque bourdeano ya que posibilita conocer y distinguir las diferentes formas de capital que están en la base de accesos y usos diferenciales de TD. Al respecto, considera que es necesario redefinir qué se entiende por acceso, dándole un lugar central al contexto de acceso que involucra no solo cuestiones relativas a los tiempos y costos para conectarse, si no también cuestiones como la privacidad, los espacios para usar las TD, los saberes, entre otros. A su vez, plantea que hay que analizar la relación entre acceso y uso, entendiendo que la falta de uso no puede explicarse únicamente a partir del acceso. Selwyn propone el concepto de capital tecnológico (Selwyn, 2004, p. 354) como un término que posibilita dar cuenta del modo en que se van acumulando y desarrollando habilidades para el uso de las tecnologías a lo largo de las trayectorias vitales de las personas.

Como podemos notar, inicialmente los interrogantes giraban en torno al acceso a dispositivos y a conectividad, y las perspectivas de abordaje se centraban en el concepto de brecha digital (Benítez Larghi y Duek, 2016). Con los años y las transformaciones ligadas a la expansión del acceso a las TD, comenzaron a ganar lugar las preguntas sobre los usos de TD (Benítez Larghi y Duek, 2016) y sobre 
las alfabetizaciones necesarias para usarlas con diversos fines; cuestión esta última que aloja una multiplicidad de definiciones aún en construcción en este campo de estudios. A continuación presentamos perspectivas que consideramos tienen gran potencialidad para abordar los procesos de configuración de desigualdades asociadas al acceso y uso de TD, teniendo en cuenta los diversos niveles en que se producen y reproducen las desigualdades y sus dimensiones materiales y simbólicas. Estas perspectivas no niegan el carácter performativo que pueden tener las condiciones de acceso a TD en las prácticas y competencias que desarrollan las personas, pero proponen situar tales condiciones en contextos sociales, económicos y culturales específicos para lograr identificar de qué manera las personas dotan de significado a las TD y cómo esto se relaciona con tramas de desigualdades.

Desde un enfoque multidimensional y relacional de las desigualdades, Reygadas (2008) propone alejarse del determinismo tecnológico que tiende a presuponer linealmente efectos de las tecnologías, poniendo el acento en los artefactos y no en el modo en que los individuos se pueden apropiar de estos, como también de visiones normativas acerca de las tecnologías y sus usos. Reygadas invita a pensar a las TD como parte de redes de desigualdad en donde se conjugan "capital informático objetivado" (Reygadas, 2008, p. 201), entendido como los elementos materiales necesarios para utilizar una computadora e Internet, con "capital informático incorporado" (Reygadas, 2008, p. 201), que comprende aquellos conocimientos y habilidades que posibilitan hacer un uso de los dispositivos tecnológicos y que implican tanto saber leer y escribir, como encontrar y ponderar información y saber utilizar software (Reygadas, 2008). En sintonía con lo mencionado por Selwyn (2004), Reygadas considera que en el desarrollo de ambos tipos de capital, pero especialmente en el segundo, tienen particular importancia los saberes y capacidades desarrollados durante distintas instancias de aprendizaje y socialización, que exceden a la educación formal y la capacitación específica e informática, y son más bien producto de trayectorias de vida en donde este tipo de formación es valorada e incentivada (Reygadas, 2008).

En la intersección entre desigualdades y usos de TD, Helsper retoma los aportes del concepto de privación relativa y propone la noción de "relatividad social de las desigualdades digitales" [social relativity of digital inequalities] (Helsper, 2017). A partir de esto, sugiere analizar cómo se configuran desigualdades en relación a las TD haciendo énfasis ya no solo en elementos a nivel estructural, ni tampoco individuales, sino en las relaciones, es decir, en los procesos a nivel grupal -y sus cambios- y de qué forma allí se construyen los vínculos con las TD y experiencias relativas a la inclusión-exclusión (Helsper, 2017). En este sentido, se vuelve importante considerar de manera contextual e intragrupal cómo se construyen deseos en torno a la posesión, usos y saberes asociados a TD. Helsper también considera que una de las contribuciones del enfoque de la privación relativa al estudio de las desigualdades y su relación con las TD se vincula con estudiar de qué manera, en los espacios que habitan cotidianamente, las personas construyen opiniones y decisiones en torno a las $\mathrm{TD}$, a la vez que en esas interacciones construyen a ciertos otros, a los similares, como referencias para compararse (Helsper, 2017). La autora propone analizar procesos grupales a partir del "principio de similaridad" de la teoría de la privación relativa (Helsper, 2017, p. 9), según el cual los individuos se evalúan a sí mismos, 
perciben su posición y posibilidades, en relación con su valoración sobre la situación de quienes consideran sus similares, más que con un juicio sobre la posición de los no similares (Helsper, 2017).

Por último, el concepto de apropiación desarrollado por Thompson (1998) para estudiar procesos comunicacionales, y recuperado por Winocur (2009) para el estudio de los modos en que individuos y grupos se vinculan con y a través de las TD, presenta también gran potencial para explorar las articulaciones entre desigualdades y tecnologías, en el marco de entramados sociales, culturales y familiares específicos (Winocur, 2009). La apropiación es pensada como un proceso tanto simbólico como material por medio del cual un sujeto o grupo toma el contenido significativo de un artefacto y lo hace propio (Thompson, 1998). La apropiación se construye sobre la base de experiencias presentes y pasadas con diversas tecnologías, así\# como de expectativas, nociones previas e ideas, tanto individuales como familiares, sobre estos artefactos (Cabrera Paz, 2009; Winocur, 2009). Las modalidades que adquiere la apropiación se vinculan también con posiciones de clase y relaciones con los otros, y se desarrollan en relación con entramados culturales particulares que le otorgan valoración y significación a los artefactos tecnológicos (Winocur, 2009). En este sentido, Winocur ha sostenido que el uso de las TD "no es la relación pragmática con un objeto, sino con el universo de representaciones culturales con las cuales esa tecnología se articula en la vida de las familias de diversa condición sociocultural" (Winocur, 2009, p. 19). Por lo tanto, debemos tomar en consideración que las representaciones y usos se constituyen en relación con la historia y las prácticas de cada grupo social y están, a su vez, mediados por los procesos de desigualdad, las construcciones de los roles de género y la clase social.

\section{Las alfabetizaciones}

Como analizamos en el apartado anterior, las primeras líneas de investigación sobre la relación entre TD y desigualdades tendían a enfocarse principalmente en el acceso a dispositivos e Internet. De acuerdo con Warschauer y Ames, de manera un tanto inocente se asumía que las desigualdades entre personas, países y regiones se esfumarían una vez asegurada la disponibilidad de aparatos y conectividad (Warschauer y Ames, 2010). Cuando se hizo evidente que las desigualdades persistían a pesar del crecimiento de los niveles de acceso a Internet y dispositivos, fueron incorporándose interrogantes sobre la calidad y diversidad del acceso, el tipo y circunstancias de uso, las prácticas, los actores que actuaban como soporte en cada contexto, para intentar comprender por qué las personas desarrollaban competencias de manera diferencial y desigual (Livingstone y Sefton-Green, 2018; Helsper, 2017; boyd, 20144; entre otros). En ese contexto comenzaron a emerger con más fuerza los interrogantes sobre los saberes y competencias necesarias para utilizar con diversos fines TD y su vinculación con distintos tipos de desigualdades.

En este punto es importante notar que, a nivel mundial, los esfuerzos de los Estados para proveer a la ciudadanía de TD y fomentar el desarrollo de conocimiento y habilidades en materia de TD tuvieron como principal destinatario al sistema educativo (Morales, 2015). En nuestro país, tanto las iniciativas llevadas adelante durante los años 90, como las de aplicación más 
reciente, han ubicado al sistema educativo como el actor clave en la ejecución de las políticas y, a su vez, como el garante del cumplimiento de sus objetivos. Esto ha generado amplias expectativas respecto de la mejora tanto de la educación como de las desigualdades (Kalman, 2017; Benítez Larghi, 2016), a la vez que ha reforzado el carácter de sobre demandado del sistema educativo argentino (Tenti Fanfani, 2010). A su vez, las TD han sido consideradas una suerte de "llave mágica" (Dussel, 2016) capaz de transformar los procesos de enseñanzaaprendizaje al interior de las escuelas. De esta forma, la ampliación del foco de indagación del acceso hacia los usos y las alfabetizaciones tuvo, y continúa teniendo, importantes resonancias en los distintos niveles y actores de los sistemas educativos.

Para comenzar, una de las discusiones que ha atravesado los estudios que indagan en los aprendizajes y los usos de TD gira en torno a los conceptos de "nativos e inmigrantes digitales" propuestos por Prensky (2001). Los términos fueron desarrollados en el marco de una reflexión acerca de las nuevas formas de vincularse con el conocimiento y el aprendizaje que tenían niños/as y jóvenes producto del uso de las TD, y los desafíos que ello acarreaba para docentes que se relacionaban con estos artefactos de una manera totalmente distinta. El principal criterio de demarcación entre nativos/as e inmigrantes digitales, y lo que será luego foco de críticas, es la generación. Mientras niños/as y jóvenes, los/ as nativos/as, según Prensky, han crecido y vivido toda su vida usando diversos tipos de TD y tienen por ese motivo amplios saberes y habilidades para operar distintos artefactos, los/as adultos/as, es decir, los/as inmigrantes, han entrado en el mundo de las TD más tarde, se han incorporado -o buscan hacerlo-pasadas las instancias de socialización que tienen lugar en la niñez y adolescencia y carecen de buena parte de los conocimientos para incorporarse en el uso de las TD (Prensky, 2001).

Los conceptos de inmigrantes y nativos digitales gozaron de gran aceptación y difusión a nivel mundial, y en nuestra región durante varios años. Durante la última década, en distintas latitudes, han emergido miradas críticas a la formulación de Prensky (Benítez Larghi, Lemus, Moguillansky y Welschinger Lascano, 2014; White y Le Cornu, 2011; Helsper y Eynon, 2009, Stoerger, 2009; Kennedy, Judd, Churchward, Gray y Krause, 2008; McKenzie, 2007), propuestas de conceptualizaciones alternativas (White y Le Cornu, 2011; y Stoerger, 2009, por ejemplo), junto con un distanciamiento de su propia caracterización en términos de nativos e inmigrantes digitales desarrollado por Prensky (2009). De acuerdo con White y Le Cornu (2011), estos conceptos tuvieron utilidad inicialmente para identificar los modos en que distintos actores se vinculaban con las TD y construir tipologías (White y Le Cornu, 2011). A su vez, las preocupaciones plasmadas por Prensky (2001) contribuyeron a motorizar las discusiones orientadas a transformar los procesos educativos a la luz de los cambios tecnológicos y desarrollar aprendizajes en entornos mediados electrónicamente (White y Le Cornu, 2011).

Más allá de estos puntos a favor, los términos contribuyeron a entender los vínculos de los individuos con las TD de una manera fija y rígida, como compartimientos estancos (White y Le Cornu, 2011; Helsper y Eynon, 2009, Stoerger, 2009). A su vez, el establecimiento a priori del criterio etario para distinguir entre uno y otro grupo fue también duramente cuestionado por 
distintos motivos. En primer lugar, por no dar cuenta del modo en que las desigualdades de clases se traducen en dificultades para el acceso a TD y/o para el desarrollo de habilidades (White y Le Cornu, 2011). En segundo lugar, por invisibilizar la existencia de saberes y capacidades heterogéneas entre los/ as jóvenes para usar las TD y tender a unificar las experiencias de la juventud con estos artefactos (White y Le Cornu, 2011; Helsper y Eynon, 2009; Stoerger, 2009). En tercer lugar, las críticas se han erigido sobre la tendencia de estos conceptos a universalizar un conjunto de "saberes deseables" para todas las personas, sin tener en cuenta que existen conocimientos y habilidades que son más o menos significativos en cada contexto y momento biográfico (White y Le Cornu, 2011). En cuarto lugar, las críticas al concepto se han dirigido contra la falta de evidencia empírica que permita sostener que los/as jóvenes tienen amplias y diversas capacidades con las TD y que los/as adultos/as carecen de estas (Stoerger, 2009).

El uso acrítico de estos conceptos y sin tener en cuenta sus implicancias sobre la relación de jóvenes y adultos/as con las TD, puede volverse especialmente problemático en la formulación de políticas públicas y en la educación, en tanto opaca, las condiciones diferenciales en las que se encuentran distintos/as jóvenes con respecto a sus habilidades y saberes para usar las TD, así como para acceder a estas (Benítez Larghi, Lemus, Moguillansky y Welschinger, 2014; Helsper y Eynon, 2009). Al mismo tiempo, dificulta la interacción entre estudiantes/ jóvenes y docentes/adultos al hacer sentir a estos últimos desprovistos de conocimientos y herramientas frente a nativos/as que son presentados como con un amplio dominio de las TD (Helsper y Eynon, 2009). La experiencia, la educación y el acceso a TD son elementos que tienen más peso para explicar por qué ciertos individuos se vuelven "nativos digitales" que la dimensión generacional en sí (Benítez Larghi, Lemus, Moguillansky y Welschinger, 2014; Helsper y Eynon, 2009).

En la última década, el interés por comprender cómo se desarrollan competencias para usar tecnologías e información digital, junto a las discusiones sobre las alfabetizaciones necesarias y deseables para estudiar, comunicarse, informarse, formar parte del mercado de trabajo y ejercer la ciudadanía en sociedades crecientemente mediatizadas, fueron ganando lugar y suscitaron diversas conceptualizaciones como alfabetización digital, alfabetización mediática, alfabetización en datos e información y multialfabetizaciones (Scolari, 2018; Palsa y Ruokamo, 2015; Meyers, Erickson y Small, 2013; Cabello, 2013; Potter, 2010). Así, nos encontramos con una pluralidad de términos que se utilizan, con diversa reflexión y adscripción teórica, para denotar los procesos de aprendizaje, adquisición de conocimientos y desarrollo de habilidades para utilizar TD. Como sostiene Buckingham (2018), designar un conjunto de competencias como resultado de una práctica y proceso de alfabetización implica una diputa por el estatus social que se le asigna a estas competencias, poniendo de relieve que se adquieren y desarrollan de manera laboriosa, a través de la práctica sistemática y no de forma natural y automática.

El concepto de alfabetización mediática ha cobrado gran protagonismo, tanto por su amplia difusión en la investigación académica sobre el tema (Asmar, van Audenhove y Mariën, 2020; Hobbs, 2010; entre otras) como porque ha poblado los diseños curriculares de distintos países (Ruokamo, 
Kotilainen, Kupiainen y Maasilta, 2016) y las recomendaciones de organismos internacionales (UNESCO, 2013). ${ }^{5}$ De acuerdo con Kotilainen y Suoninen (2013), a nivel internacional, la alfabetización mediática e informacional hace referencia al desarrollo de capacidades para usar medios para hallar información y comunicarse con otras personas, para expresarse y para analizar críticamente la calidad de la información. Sin embargo, es necesario identificar cuáles son los significados particulares que adquieren las tecnologías y medios digitales en cada contexto cultural, así como en qué aspectos se necesita y/o desea hacer énfasis a través del aprendizaje (Ruokamo, Kotilainen, Kupiainen y Maasilta, 2016). Es decir, la alfabetización mediática adquiere diversos contenidos en cada contexto, manteniendo siempre el objetivo de desarrollar análisis y prácticas críticas con respecto a los contenidos que consumen a través de los medios.

Por su parte, la noción de alfabetización digital también ha recibido vasta atención y se propone como un concepto relacionado a otras alfabetizaciones, como la mediática y la tradicional. La alfabetización digital refiere tanto a las habilidades técnicas para operar tecnologías digitales como a la capacidad para evaluar y utilizar el conocimiento adquirido a través de la participación en entornos digitales (Ilomäki, Taalas y Lakkala, 2012; Kotilainen y ArnoldsGranlund, 2010). Involucra, por tanto, una combinación de habilidades técnicasprocedimentales, cognitivas y socioemocionales (Aviram y Eshet-Alkalai citados en Ilomäki, Taalas y Lakkala, 2012). A nivel local, este concepto ha ganado mayor lugar que otros en las publicaciones académicas (Pini y Mihal, 2017; Cabello, 2013) y también en las recientes reformas de diseños curriculares. ${ }^{6}$ En relación con esto, la propuesta de Cabello (2013) para estudiar las competencias digitales propone una mirada amplia e integral de la alfabetización digital y considera necesario tener en cuenta las siguientes dimensiones: 1) instrumental (vinculada a la capacidad técnica de usar una TD); 2) cognitiva (refiere al desarrollo de saberes y habilidades para la búsqueda, selección, jerarquización, análisis, comprensión y producción de información a través de TD;3) actitudinal (vinculada a valoraciones, significados atribuidos a las TD y las actitudes en relación a estos); 4) política (concerniente al desarrollo de un posicionamiento respecto de las TD y sus implicancias) y 5) comunicativa (asociada a la producción de contenidos, la expresión y la comunicación con otras personas) (Cabello, 2013, p. 25).

Sefton-Green, Nixon y Erstad (2009) destacan la potencialidad del concepto para explicar cómo las personas aprenden y se involucran con las TD y de qué modos estas median en nuestras múltiples interacciones sociales. Sin embargo, señalan que es necesario analizar el desarrollo de la alfabetización digital en su encuentro con espacios y prácticas de aprendizaje formal e informal y no circunscribirla únicamente al aprendizaje escolar.

De acuerdo con Scolari, las principales diferencias entre el alfabetismo transmedia, el mediático, el digital y el alfabetismo que podríamos denominar "tradicional", están dadas por el tipo de lenguaje, los soportes, los objetivos y las direcciones en que se desarrollan (Scolari, 2018). Mientras que el alfabetismo "tradicional" promueve la lectura y escritura a partir de libros y textos impresos, promoviendo lectores/as y escritores/as críticos/as en un sentido de arriba hacia abajo (de los espacios formales a los informales), el alfabetismo mediático promueve lenguajes multimodales, tiene de soportes a los medios impresos y 
audiovisuales, busca desarrollar espectadores/as críticos/as que, eventualmente, actúen como productores/as, y se orienta tanto de abajo hacia arriba como viceversa. Por su parte, el alfabetismo transmedia busca construir lenguajes multimodales, apoyándose en redes digitales y medios interactivos y transmedia, para desarrollar prosumidores/as críticos/as, principalmente en un sentido de abajo hacia arriba, es decir, partiendo de las prácticas informales (Scolari, 2018).

El concepto de alfabetización transmedia, propuesto por Scolari (2018), se plantea como una instancia que dialoga e integra en cierta medida a las demás alfabetizaciones y refiere a las "habilidades, prácticas, prioridades, sensibilidades, estrategias de aprendizaje y formas de compartir que se desarrollan y se aplican en el contexto de las nuevas culturas participativas" (Scolari, 2018, p. 17) y que tienen como resultado el desarrollo de competencias que permiten usar TD y moverse en un ecosistema de medios dinámico. El concepto busca poner de relieve modalidades de aprendizaje basadas en la resolución de problemas, el auto aprendizaje y en la experimentación (Scolari, 2018), así como dar cuenta del carácter ubicuo de las prácticas de aprendizaje en TD.

A nivel mundial, la ausencia de un diálogo entre las prácticas de aprendizaje en medios y tecnologías que tienen lugar en las escuelas y en la vida cotidiana ha empezado a ser una preocupación cada vez más acuciante (Buckingham, 2018). A diferencia de nociones desarrolladas previamente, el alfabetismo transmedia presupone que las personas no solo consumen, sino que también producen contenidos e información y aprenden a vincularse con las TD en espacios formales (aunque principalmente en instancias informales). En relación a esto último, radica una de las potencialidades del concepto, que permite identificar y caracterizar las modalidades en que las generaciones más jóvenes están desarrollando competencias transmedia en un ida y vuelta entre la escuela, el hogar, el grupo de amigos/as pero también YouTube, las redes sociales y los blogs (Scolari, 2018). En un contexto en donde la demanda por incorporación de TD en la enseñanza formal tiene larga data (Morales, 2015), esta propuesta teórica puede resultar productiva para abordar el marcado desajuste entre las dinámicas que organizan la producción y circulación de conocimientos en las aulas y fuera de ellas (Peirone, 2018).

Como analizamos al comienzo, distintos tipos de desigualdades permean y dan forma a los procesos de apropiación de las TD y al desarrollo de alfabetismos transmedia. Los diversos soportes sociales (Martuccelli, 2007) con los que cuentan las personas, como las instituciones educativas y los programas de alfabetización transmedia, la familia y el grupo de amigos/as, han sido destacados como aspectos clave para la promoción de competencias, poniendo en evidencia que las desigualdades no solo se explican por el acceso a las TD (Asmar, van Audenhove y Mariën, 2020). En este misma dirección, la alfabetización transmedia que tiene lugar al interior de algunos hogares (con diversa modalidad y características) se suma a la lista de prácticas que se despliegan en las familias para sostener y acrecentar su posición social (Lemus, 2020, a nivel nacional y Livingstone y Sefton-Green, 2018, para el caso inglés, entre otros). 


\section{Conclusiones}

Las reflexiones acerca del vínculo entre TD y desigualdades se han constituido en un tema central en la agenda política y académica. La ampliación del foco de indagación, del acceso a las TD al desarrollo de alfabetizaciones, no niega las desigualdades asociadas a la posibilidad de contar con TD en el hogar, sino que las incorpora conjuntamente con otros factores de peso para usar tecnologías con fines diversos y hacer frente a las demandas del mercado de trabajo y de la formación académica. En un contexto nacional en donde el acceso hogareño a computadoras alcanza al 60,90\%, a Internet al 82,90\%, y el 84,30 \% de la población mayor de 4 años utiliza teléfono celular (INDEC, 2020), la pregunta por el acceso no se agota sino que se reconfigura y nos lleva a preguntarnos por la calidad y diversidad de las TD presentes en los hogares, por los factores que influyen en el desarrollo de competencias transmedia, así como por los soportes con los que cuentan niños/as, jóvenes y adultos/as para aprender a usar TD.

Las medidas de aislamiento y distanciamiento social preventivo y obligatorio tomadas por la pandemia de COVID19, y la consecuente puesta en marcha de dispositivos de enseñanza-aprendizaje a distancia y virtuales han puesto de relieve -una vez más- que la apropiación de las TD y el desarrollo de competencias transmedia requiere del acceso a dispositivos y conexiones de calidad en donde la individualización del acceso, es decir, contar con un dispositivo propio se vuelve clave para sostener y potenciar las prácticas de enseñanza-aprendizaje y demanda de un entorno (amigos, familia, escuela) que acompañe y potencie la experimentación con TD. Indagar en los soportes intersubjetivos, en las prácticas, significados y motivaciones involucradas en el desarrollo de competencias transmedia, permitirá profundizar en la comprensión de las diversas y cambiantes articulaciones entre desigualdades y tecnologías digitales.

\section{Referencias}

Asmar, A., van Audenhove, L. \& Mariën, I. (2020). Social Support for Digital Inclusion: Towards a Typology of Social Support Patterns. Social Inclusion, 8(2), 138-150. https://doi.org/10.17645/si.v8i2.2627

Barrantes, R. (2007). Análisis de la demanda por TICs: ¿Qué es y cómo medir la pobreza digital? Recuperado de DIRSI-IDRC: http://www.dirsi.net/files/02-Barrantes_ esp_web_18set.pdf

Benítez Larghi, S., Aguerre, C., Calamari, M., Fontecoba, A., Moguillansky, M. y Ponce de León, J. (2012). Juventud, Sectores populares y TIC en la Argentina. Versión. Estudios de Comunicación, Política y Cultura, 27, 1-24. Recuperado de: http://bi di.xoc.uam.mx/MostrarPDF.php

Benítez Larghi, S., Lemus, M., Moguillansky, M. y Welschinger Lascano, N. (2014). Más allá del tecnologicismo, más acá del miserabilismo digital. Procesos de co-construcción de las desigualdades sociales y digitales en la Argentina contemporánea. Ensambles, 1, 57-81. Recuperado de: http://www.revistaensamb les.com.ar/ojs-2.4.1/index.php/ensambles/article/view/18/13

Benítez Larghi, S. (2016). Elogio de un "fracaso". Revista Argentina de Estudios de Juventud, 10, 1-15. https://doi.org/10.24215/18524907e011 
Benítez Larghi, S. y Duek, C. (2016). Uso y apropiación de Tecnologías de la Información y de la Comunicación. Una aproximación a su investigación en la Argentina. En M. Grillo, V. Papalini y S. Benítez Larghi (Coords.), Estudios sobre consumos culturales en la Argentina contemporánea (pp. 209-236). Ciudad Autónoma de Buenos Aires: CLACSO, CODESOC.

boyd, d. m. (2014). It's complicated. The social lives of networked teens [Es complicado. La vida social de jóvenes conectados]. New Haven, London: Yale University Press.

Buckingham, D. (2018). Prólogo. En C. Scolari (Ed.), Adolescentes, medios de comunicación y culturas colaborativas. Aprovechando las competencias transmedia de los jóvenes en el aula (pp.5-8). Barcelona: Universitat Pompeu Fabra.

Cabello, R. (2013). Migraciones digitales. Hacia un plan institucional de alfabetización digital. En R. Cabello (Coord.), Migraciones digitales: comunicación, educación $y$ tecnologías digitales interactivas (pp. 13-48). Los Polvorines: Universidad Nacional de General Sarmiento.

Cabrera Paz, J. (2009). Profesores Analógicos, Estudiantes Digitales: Una tensión cultural para la gestión pública de las TIC en Educación. Trabajo presentado en $3^{a}$ Conferencia de ACORN-REDECOM, Red Americana de Investigación en Información y Comunicación, Ciudad de México. Recuperado de: http://www.ac orn-redecom.org/papers/ProceedingsAcornRedecom2009.pdf

Camacho, K. (2005). La Brecha digital. En A. Ambrosi, V. Peugeot y D. Pimienta (Coords.), Palabras en Juego: Enfoques Multiculturales sobre las Sociedades de la Información. C\&F Éditions. Recuperado de: https://vecam.org/archives/article5 50.html

Castells, M. (2008). La era de la información: Economía, sociedad y cultura. Ciudad de México: Siglo Veintiuno Editores.

Di Maggio, P., Hargittai, E., Celeste, C., \& Shafer, S. (2001). From Unequal Access to Differentiated Use: A Literature Review and Agenda for Research on Digital Inequality. Russel Sage Foundation. Recuperado de: http://socinfogroup2.pbwo rks.com/w/file/fetch/41364119/fromunequalaccesstodifferentiateduse.pdf

Dirección General de Cultura y Educación de la Provincia de Buenos Aires (2018). Diseño curricular para la Educación Primaria: primer ciclo y segundo ciclo. La Plata: Dirección General de Cultura y Educación de la Provincia de Buenos Aires. Recuperado de: http://abc.gob.ar/primaria/sites/default/files/resolucion_ndeg_ 1482_17_diseno_curricular.pdf

Dirección General de Cultura y Educación de la Provincia de Buenos Aires. (2019). Diseño curricular para la Educación Inicial: segundo ciclo. La Plata: Dirección General de Cultura y Educación de la Provincia de Buenos Aires, 2019. Recuperado de: http://abc.gob.ar/inicial/sites/default/files/educacion_inicial_2 019.pdf

Dussel, I. (2016). Perspectivas, tensiones y límites en la evaluación de las políticas Uno a Uno en América Latina. En S. Benítez Larghi y R. Winocur Iparraguirre (Coords.), Inclusión digital: una mirada crítica sobre la evaluación del modelo Uno a Uno en Latinoamérica (pp. 145-165). Ciudad Autónoma de Buenos Aires: Teseo.

Helsper, E. \& Eynon, R. (2009). Digital natives: where is the evidence?. British educational research journal, 36(3), 503-520. Recuperado de: http://www.jstor.o $\mathrm{rg} /$ stable/27823621

Helsper, E. J. (2017). The Social Relativity of Digital Exclusion: Applying Relative Deprivation Theory to Digital Inequalities. Communication Theory, 27(3), 223-242. https://doi.org/10.1111/comt.12110 
Hobbs, R. (2010). Digital and Media Literacy: A Plan of Action (Communications and Society Program). The Aspen Institute Communications and Society Program. Recuperado de: https://www.aspeninstitute.org/wp-content/uploads/2010/11/ Digital_and_Media_Literacy.pdf

Ilomäki, L.; Taalas, P. \& Lakkala, M. (2012). Learning environment and digital literacy: A mismatch or a possibility from Finnish teachers' and students' perspective. En P. P. Trifonas, Learning the virtual life: Public pedagogy in a digital world (pp. 63-78). London/New York: Routledge.

INDEC (2020). Accesos a internet. Primer trimestre de 2020 (Informes te\#cnicos / Vol. 4, n 101. Servicios. Vol. 4, n7). Recuperado de: https://www.indec.gob.ar/u ploads/informesdeprensa/internet_06_20E1822227C2.pdf

Kalman, J. (2017). Ampliar la mirada: la evaluación de proyectos de incorporación de tecnologías digitales en contextos educativos. En S. Benítez Larghi y R. Winocur Iparraguirre (Coords.), Inclusión digital: una mirada critica sobre la evaluación del modelo Uno a Uno en Latinoamérica (pp. 167-194). Ciudad Autónoma de Buenos Aires: Teseo.

Kennedy, G. E., Judd, T. S., Churchward, A., Gray, K. \& Krause, K.L. (2008). First year students' experiences with technology: Are they really digital natives? Australasian Journal of Educational Technology, 24(1), 108-122. Recuperado de: https://pdfs. semanticscholar.org/0f3f/e5039d379b337719cb5d860b0ff32dc00a2d.pdf

Kessler, G. (2014). Controversias sobre la desigualdad. Argentina, 2003-2013. Buenos Aires: Fondo de Cultura Económica.

Kotilainen, S. \& Arnolds-Granlund, S. B. (Eds.) (2010). Media literacy education. Nordic Perspectives. Gotemburgo: NORDICOM.

Kotilainen, S. \& Suoninen, A. (2013). Cultures of media and information literacies among the young. South-north viewpoints. En U. Carlsson \& S. H. Culver (Eds.), MILID yearbook 2013: Media and information literacy and intercultural dialogue (pp. 141-162). Gotemburgo: NORDICOM.

Lamont, M., Beljean, S. \& Clair, M (2014). What is missing? Cultural processes and causal pathways to inequality. Socio-Economic Review, Advance Access, 12(3), 573-608. Recuperado de: https://scholar.harvard.edu/files/lamont/files/socioec on_rev-2014-lamont-ser-mwu011.pdf

Lemus, M. (2018). Articulaciones entre desigualdades y tecnologías digitales, un estudio de las trayectorias de vida de jóvenes de clases medias altas, La Plata 2012-2017 (Tesis doctoral). Recuperada de Memoria académica: http://www.memoria.fahce.unlp. edu.ar $/$ library? $\mathrm{a}=\mathrm{d} \& \mathrm{c}=$ tesis $\& \mathrm{~d}=\mathrm{Jte} 1741$

Lemus, M. (2020). Simuladores/as, motivados/as y autodidactas: jóvenes y aprendizajes en tecnologías digitales. Propuesta Educativa, 29(53), 86-98. Recuperado de: http://propuestaeducativa.flacso.org.ar/revista/articulos/simuladores-as-mo tivados-as-y-autodidactas-jovenes-y-aprendizajes-en-tecnologias-digitales/

Livingstone, S. y Sefton-Green, J. (2018). Class in "The Class": conservative, competitive and (dis)connected. En J. Deery \& A. Press (Eds.), Media and class: TV, film, and digital culture (pp. 176-188). New York: Routledge.

Martuccelli, D. (2007). Gramáticas del individuo. Buenos Aires: Losada.

McKenzie, J. (2007). Digital nativism, digital delusions, and digital deprivation. From Now On: the educational technology journal, 17(2). https://doi.org/10.2190/EC 38.3.a

Meyers, E., I. Erickson \& R. Small (2013). Digital Literacy and Informal Learning Environments: An Introduction. Learning, Media and Technology, 38(4), 355-367. https://doi.org/10.1080/17439884.2013.783597 
Morales, S. (2015). La apropiación tecno-media\#tica: acciones y desafíos de las políticas públicas en educación. En S. Lago Martínez (Coord.), De tecnologías digitales, educación formal y politicas públicas. Aportes al debate (pp. 27-52). Buenos Aires: Teseo.

Palsa, L. \& Ruokamo, H. (2015). Behind the concepts of multiliteracies and media literacy in the renewed Finnish core curriculum: A systematic literature review of peer-reviewed research. Seminar.net - International journal of media, technology and lifelong learning, 11(2), 101-119. Recuperado de: https://journals.oslomet.n o/index.php/seminar/article/view/2354

Peirone, F. (2018). El saber tecnológico. De saber experto a experiencia social. Virtualidad, Educación y Ciencia, 10(18), 66-80. Recuperado de: https://revistas .unc.edu.ar/index.php/vesc/article/view/23043

Pini, M. y Mihal, I. (2017). Alfabetización digital y política educativa democrática en dos normativas de Argentina. Diálogos de la comunicación, 93, 1-19. Recuperado de: https://ri.conicet.gov.ar/handle/11336/77103

Potter, A. B. (2007). Zones of silence: A framework beyond the digital divide. First Monday, 11(5). https://doi.org/10.5210/fm.v11i5.1327

Potter, W. J. (2010). The State of Media Literacy. Journal of Broadcasting \& Electronic Media, 54(4), 675-696. https://doi.org/10.1080/08838151.2011.521462

Prensky, M. (2001). Digital Natives, Digital Immigrants. On the Horizon, 9(5). Recuperado de: https://www.marcprensky.com/writing/Prensky\%20\%20Digital\%20Natives,\%20Digital\%20Immigrants\%20-\%20Part1.pdf

Prensky, M. (2009). H. Sapiens Digital: From Digital Immigrants and Digital Natives to Digital Wisdom. En Innovate: Journal of Online Education, 5(3). Recuperado de: https://nsuworks.nova.edu/cgi/viewcontent.cgi?article=1020\&context=inn ovate

Reygadas, L. (2008). La Apropiación. Destejiendo las redes de la desigualdad. México: Anthropos Editorial.

Ruokamo, H.; Kotilainen, S.; Kupiainen, R. \& Maasilta, M. (2016). Media Education Today and Tomorrow. The finnish society on media education. Recuperado de: https://mediakasvatus.fi/wp-content/uploads/2019/09/mediaeducationtod ayandtomorrow.pdf

Saraví, G. (2015). De la desigualdad a la fragmentación. En G. Saraví, Juventudes Fragmentadas. Socialización, Clase y Cultura en la Construcción de la Desigualdad (pp. 25-56). México: FLACSO.

Scolari, C. (2018). Estrategias de aprendizaje informal. En C. Scolari (Ed.), Adolescentes, medios de comunicación y culturas colaborativas. Aprovechando las competencias transmedia de los jóvenes en el aula (pp. 13-23). Barcelona: Universitat Pompeu Fabra.

Sefton-Green, J., Nixon, H. \& Erstad, O. (2009). Reviewing approaches and perspectives on "digital literacy". Pedagogies: an international journal, 4(2), 107-125. https://doi.org/10.1080/15544800902741556

Selwyn, N. (2004). Reconsidering Political and Popular Understandings of the Digital Divide. New Media and Society, 6(3), 341-362. https://doi.org/10.1177/146144 4804042519

Stoerger, S. (2009). The digital melting pot: Bridging the digital native-immigrant divide. First Monday, 14(7). https://doi.org/10.5210/fm.v14i7.2474

Tenti Fanfani, E. (2010). Aportes para el desarrollo curricular. Sociología de la educación. Buenos Aires: Ministerio de Educación. 
Thompson, J. (1998). Los media y la modernidad. Barcelona: Paidós.

UNESCO (2013). Global Media and Information Literacy Assessment Framework: country readiness and competencies [Marco global de evaluación de la alfabetización mediática e informacional: disponibilidad y competencias de los países]. Paris: UNESCO. Recuperado de: https://unesdoc.unesco.org/ark:/48223/pf0000224 655

UNESCO (2018). A global framework of reference on digital literacy skills for indicator 4.4.2 (Information Paper $N^{\circ}$ 51, UIS/2018/ICT/IP/51). Recuperado de UNESCO: http://uis.unesco.org/sites/default/files/documents/ip51-globalframework-reference-digital-literacy-skills-2018-en.pdf

Warschauer, M. (2002). Reconceptualizing the Digital Divide. First Monday, 7(7). Recuperado de: https://firstmonday.org/article/view/967/888

Warschauer, M. \& Ames, M. (2010). Can One Laptop Per Child save world's poor? Journal of International Affairs, 64(1), 33-51. Recuperado de: https://www.jstor .org/stable/24385184

White, D. S. \& Le Cornu, A. (2011). Visitors and Residents: A new typology for online engagement. First Monday, 16(9). Recuperado de: https://firstmonday.org/artic le/view/3171/3049

Winocur, R. (2007).Apropiación de la computadora e Internet en los sectores populares urbanos. Versión, 19, 191-216. Recuperado de: https://versionojs.xoc.uam.mx/in dex.php/version/article/view/299/298

Winocur, R. (2009). Robinson Crusoe ya tiene celular: la conexión como espacio de control de la incertidumbre. México: Siglo XXI.

Zukerfeld, M. (2006). Bienes Informacionales y Capitalismo Cognitivo. Conocimiento, información y acceso en el siglo XXI. Razón y palabra, 53. Recuperado de: http://www.razonypalabra.org.mx/anteriores/n54/mzukerfeld. html

\section{Notas}

1 Este artículo se ha elaborado en el contexto de la Red INCASI, proyecto europeo que ha recibido financiación del programa de investigación H2020 de la Unión Europea, Marie Skłodowska-Curie GA No 691004, coordinado por el Dr. Pedro López-Roldán. El artículo refleja la opinión de la autora y la Agencia no es responsable del uso que se pueda hacer de la información que contiene.

2 En este artículo, con "tecnologías digitales" nos referimos a computadoras (de escritorio, notebooks, netbooks), tablets, smart phones, e Internet, en tanto tecnologías que "procesan, transmiten, almacenan o generan Información Digital" (Zukerfeld, 2006, s/d).

3 Para un análisis en detalle de la evolución del acceso a TD a nivel mundial, regional y argentino, se sugiere revisar Lemus (2018).

4 A lo largo del artículo el apellido boyd aparece con minúscula inicial, respetando el posicionamiento de la autora respecto de las letras capitales: http://www.danah.org/n ame.html (Recuperado el 12/06/2018).

5 Por ejemplo, anualmente UNESCO organiza la "Media and Information Literacy Week" con el objetivo de promover la alfabetización en medios e información entre niños/as y jóvenes de distintas partes del mundo.

6 El fomento a la alfabetización digital cobró protagonismo en el Diseño Curricular para la Educación Inicial (2019) y la Educación Primaria (2018) de la Dirección General de Cultura y Educación de la Provincia de Buenos Aires (en adelante, DGCyE) (DGCyE, 2019 y 2018). Allí se prescriben una serie de contenidos que deben ser enseñados de forma transversal en todas las áreas (DGCyE, 2019 y 2018). 